\title{
Sulfonamide resistance in a disseminated infection caused by Nocardia wallacei: a case report
}

\author{
Nadim Cassir ${ }^{1,2}$, Matthieu Million ${ }^{1,2}$, Remy Noudel ${ }^{3}$, Michel Drancourt ${ }^{1}$ and Philippe Brouqui ${ }^{1,2,4^{*}}$
}

\begin{abstract}
Introduction: Nocardial infections, although rare, are challenging for clinicians to treat. Recent contradictory reports of sulfonamide resistance have raised concerns about using this drug to treat nocardial infections.

Case presentation: A 62-year-old immunocompetent Caucasian woman showed disseminated pulmonary nodules and a brain abscess by chest computed tomography and brain magnetic resonance imaging, respectively.

Multidrug-resistant Nocardia wallacei was cultured from a stereotactic brain biopsy and confirmed by $16 \mathrm{~S}$ ribosomal ribonucleic acid gene sequencing. After the first-line treatment failed, a long course of trimethoprim-sulfamethoxazole was prescribed with no evidence of recurrence. To the best of our knowledge, this is the first report of a Nocardia wallacei disseminated infection in an immunocompetent patient, and it is the first detailed description of successful treatment with trimethoprim-sulfamethoxazole despite the resistance observed in vitro.

Conclusion: Species identification of clinical isolates is critical for diagnosis, a prediction of antimicrobial susceptibility and epidemiological tracking. In the case of Nocardia wallacei, the clinical outcome suggests that sulfonamides can be used for treatment despite ambiguous results from in vitro susceptibility tests.
\end{abstract}

Keywords: Neurosurgery, Nocardia wallacei, Sulfonamide resistance

\section{Introduction}

Nocardia species are aerobic, Gram-positive bacteria of the order Actinomycetales. They are ubiquitous in the environment and live in soil, organic matter and water. They have been recognized as opportunistic human pathogens, and infection with Nocardia species usually occurs through inhalation or direct cutaneous inoculation. Recently, an increased number of nocardial infections, including brain abscess, have been reported in individuals without significant immunosuppression [1].

To date, more than 50 Nocardia species have been isolated from clinical infections, and our knowledge of new species and taxonomic relationships is constantly expanding [2]. Pijper and Pullinger reported the first

\footnotetext{
*Correspondence: philippe.brouqui@univmed.fr

${ }^{1}$ Unité de Recherchesur les Maladies InfectieusesTropicales et Emergentes

(URMITE), UM63, CNRS 7278, IRD 198, Inserm 1095,

InstitutHospitalo-UniversitaireMéditerranée-Infection, Aix-Marseille-Université,

Marseille, France

${ }^{2}$ Service de Maladies Infectieuses et Tropicales, Hôpital Nord, Assistance

Publique-Hôpitaux de Marseille, Marseille, France

Full list of author information is available at the end of the article
}

instance of a $N$. transvalensis infection in 1927 after identifying it as the causative agent of a mycetoma of the foot in a South African patient [3]. In 1997, Wilson et al. found that the drug pattern type IV of $N$. asteroides was more closely related to $N$. transvalensis than to other members of the $N$. asteroides complex, according to their biochemical characteristics, molecular differences and antimicrobial susceptibilities [4]. In 2008, Conville et al. proposed that $N$. asteroides drug pattern IV be given the new species designation of $N$. wallacei [5]. To the best of our knowledge, our present report is the first case of disseminated infection caused by $N$. wallacei in an immunocompetent patient.

Recent sulfonamide susceptibility tests of $N$. wallacei isolates in different laboratories gave contradictory results, even when they were performed in duplicate [6]. This variability raises questions regarding the accurate interpretation of these susceptibility tests in clinical settings. On the one hand, there have been recent alarming reports of increased in vitro sulfonamide resistance in Nocardia species [7-9], but on the other hand, the sulfonamide

\section{() Biomed Central}


antibiotic combination of trimethoprim-sulfamethoxazole (TMP-SMX) remains one of the first-choice drugs for treating nocardiosis [1]. In this case report, we present a patient who was successfully treated for a disseminated infection caused by $N$. wallacei with a long course of TMP-SMX after a first-line antibiotic therapy failure. Initial antibiotic susceptibility tests showed that the infecting organism was resistant to multiple drugs, including sulfonamides. To the best of our knowledge, this is the first case report that clearly describes the successful treatment of a disseminated infection caused by $N$. wallacei with sulfonamides despite an initial in vitro resistance.

\section{Case presentation}

A 62-year-old Caucasian woman living in an urban area of Corsica was admitted to our hospital with an increasing headache, breathing failure and pleuritic chest pain on her right side. She had no relevant past medical history other than pneumonia 6 months ago. Because of the persistence of dyspnea and radiographic findings consistent with a recurrence of infection, she underwent a fibroscopic bronchoalveolar lavage 1 month before admission. The culture grew Nocardia species, and the susceptibility test results were pending at the time of admission. At admission, the patient was receiving a treatment of ceftriaxone (1g) and amikacin (900 mg) once a day intravenously for 15 days. Her temperature was $38.2^{\circ} \mathrm{C}$ with $89 \%$ oxygen saturation in ambient air. Her physical and neurological examinations were unremarkable. Laboratory analyses yielded the following numbers: hemoglobin concentration, $11.8 \mathrm{~g} / \mathrm{dL}$; a white blood cell count of 4 cells $\times 10^{9} / \mathrm{L}$ (79\% neutrophils, $8 \%$ lymphocytes); creatinine concentration, $0.68 \mathrm{mg} / \mathrm{dL}$ and moderately elevated C-reactive protein $(62 \mathrm{mg} / \mathrm{L}$; normal is less than $10 \mathrm{mg} / \mathrm{L}$ ). Blood cultures and a human immunodeficiency virus test were all negative. Chest computed tomography $(\mathrm{CT})$ revealed an interstitial distribution of multiple pulmonary nodules, fluid in the major fissure and a small pleural effusion. CT and magnetic resonance imaging (MRI) brain scans conducted upon arrival showed three nodular lesions in the left frontoparietal lobe surrounded by perilesional edema with a faint ring enhancement after contrast injection. The hyper-intense appearance of the lesions in diffusion-weighted images of the MRI scan was highly suggestive of a brain abscess. Stereotactic brain biopsy of the lesions identified a modified acid-fast variable branching filamentous bacterium that had morphology consistent with Nocardia species. The final Nocardia species culture was obtained by inoculating a Columbia agar plate containing $5 \%$ sheep blood (bioMérieux) with pus from the abscess. The culture was incubated at $37^{\circ} \mathrm{C}$ in a $5 \%$ carbon dioxide atmosphere. The definite identification was based on the partial sequencing of the $16 \mathrm{~S}$ ribosomal ribonucleic acid gene (over a length of 1439 nucleotides) as previously described [10]. The sequencing results showed a $100 \%$ sequence similarity with the $N$. wallacei reference sequence (GenBank accession number GQ853074.1). No other organisms were isolated from the patient sample. The antibiotic susceptibility of the isolate was assessed on Mueller-Hinton agar (bioMérieux) using the disk method (Mast Diagnostics). The isolate was determined to be susceptible to ceftriaxone, ciprofloxacin and linezolid and resistant to amikacin, co-trimoxazole and imipenem. These results were consistent with susceptibility testing performed at the hospital of origin using the disk method. According to the recommendations of the Clinical and Laboratory Standards Institute for the susceptibility testing of Nocardia species, an additional antimicrobial test was performed by the microdilution method, and this test indicated that the isolate was susceptible to co-trimoxazole (minimum inhibitory concentration $(\mathrm{MIC})<2 / 38 \mathrm{mg} / \mathrm{L})$ [11]. After admission, the patient received treatment with imipenem ( $1 \mathrm{~g} / 8$ hours) and amikacin $(900 \mathrm{mg} / 24$ hours) and was switched to linezolid (600mg/12 hours) and ciprofloxacin (500mg/8 hours) after receiving the susceptibility test results. Two weeks later, because of the appearance of neurological signs such as Broca's aphasia and the extension of the cerebral abscess while on treatment, a new surgical drainage was performed by open craniotomy excision. The antibiotic regimen was switched to TMPSMX (2400mg/day-480mg/day) and linezolid (600mg/12 hours). The linezolid was stopped after a total of 4 weeks to limit the risk of hematological toxicity. The neurological signs were improved, and the biological parameters were normalized after 1 month of treatment. Six months later, the patient presented with an isolated neutropenia and concomitant skin rash consistent with a hematological toxicity of the TMP-SMX. The treatment was then switched to moxifloxacin $(400 \mathrm{mg} / 24$ hours, administered orally) for the remaining 6 months of the treatment period. A 1-year followup after the antibiotic regimen ceased showed no evidence of a recurrent infection.

\section{Discussion}

Although Nocardia species are a rare cause of intracranial abscess and represent only $1 \%$ to $2 \%$ of all cerebral abscesses, its mortality rate $(31 \%)$ is considerably greater than that of other agents that can cause cerebral abscesses $(<10 \%)$ [12]. In this context, an accurate diagnosis and early treatment are challenging. Initial combination therapy with two active agents is recommended for patients with disseminated or severe nocardiosis [1]. Empirical therapy should consider the local ecology (e.g., particular strains, trends in antimicrobial susceptibility) and be adapted after the antibiotic susceptibility testing. However, these susceptibility tests may not always be reliable, as 
shown by the contradictory results of TMP-SMX susceptibility trends. Uhde et al. reported that among 765 Nocardia isolates submitted to the American Center for Disease Control and Prevention in Atlanta, Georgia from 1995 to 2004, 61\% were resistant to SMX, and $42 \%$ were resistant to TMP-SMX [7]. In a 2011 study of 186 Nocardia species isolated from patients in Spain, Larruskain et al. reported that $16.1 \%$ were resistant to TMP-SMX [8]. A recent report from Canada stated that $43 \%$ of the 157 of Nocardia isolates recovered from Quebec (1988 to 2008) were resistant to TMP-SMX [9]. By contrast, other researchers have reported a low incidence of resistance to TMP-SMX. Brown-Elliot et al. stated that among 552 susceptibility results submitted for review, only 14 isolates (2.5\%) were identified as sulfonamide-resistant, with three isolates resistant to TMP-SMX and 11 resistant to SMX [13]. These results are strikingly similar to the low incidence $(2 \%)$ of sulfonamide resistance observed by Lai $e t a l$. in a study of Nocardia isolates in Taiwan [14]. The disparity among the reported data, even in the same country, is of interest because it suggests that factors other than geographic location may play a major role in the susceptibility patterns reported; the most probable factor is a difference in methodology or interpretation. The exchange of organisms between two or more accredited laboratories has been proposed as a way to enhance the accuracy of susceptibility testing [13]. Despite this recommendation, a recent multisite study by Conville et al. found that there was a lack of reproducibility for the sulfonamide MIC results, especially when testing $N$. wallacei and even within a particular reference laboratory [6]. Among the reasons for this variability discussed by the authors was the difficulty associated with obtaining a homogeneous organism suspension or inoculum because of the clumping ability of Nocardia species. Of interest, this study indicated that the disk diffusion test for sulfonamides was useful for validating the MIC results obtained by broth microdilution. In our case, the results of these two susceptibility tests were contradictory for sulfonamides.

$N$. wallacei is expected to be susceptible to TMP-SMX and resistant to amikacin [6]. Isolates reported to be resistant to sulfonamides have nonetheless been successfully treated with sulfonamides [9]. However, rare clinical reports have described the treatment failure of Nocardia with TMP-SMX [15]. In the present case, the clinical response to TMP-SMX was discordant with the results of the initial in vitro susceptibility test. Recently, large microbiological studies have raised concerns regarding the limits of interpretation of the TMX-SMX susceptibility test results. This is the first case of disseminated infection caused by $N$. wallacei that demonstrates the conflict between the in vitro resistance to sulfonamides and the clinical response to TMP-SMX.

\section{Conclusion}

We consider it necessary to preserve the traditional approach to empirical therapy for infections involving Nocardia species, and this therapy almost always includes TMP-SMX. One must consider the inherent difficulties of interpreting MIC results when evaluating reports of resistance to TMP-SMX. In the present case, the clinical outcome and species identification guided the treatment irrespective of the results of the susceptibility test.

\section{Consent}

Written informed consent was obtained from the patient for the publication of this case report. A copy of the written consent is available for review by the Editor-in-Chief of this journal.

\section{Competing interests}

The authors declare that they have no competing interests.

\section{Authors' contributions}

NC took care of the patient and analyzed and interpreted the patient data. MM and RN took care of the patient. MD helped perform the microbiological investigations. PB took care of the patient and was a major contributor in writing the manuscript. All authors read and approved the final manuscript.

\section{Author details}

'Unité de Recherchesur les Maladies InfectieusesTropicales et Emergentes (URMITE), UM63, CNRS 7278, IRD 198, Inserm 1095,

InstitutHospitalo-UniversitaireMéditerranée-Infection, Aix-Marseille-Université, Marseille, France. ${ }^{2}$ Service de Maladies Infectieuses et Tropicales, Hôpital Nord, Assistance Publique-Hôpitaux de Marseille, Marseille, France. ${ }^{3}$ Service de Neurochirurgie, Hôpital Nord, Assistance Publique-Hôpitaux de Marseille, Marseille, France. ${ }^{4}$ InstitutHospitalo-Universitaire en Maladies Infectieuses et Tropicales, Hôpital Nord, AP-HM, Chemin des Bourrelys, 13915, Marseille cedex 20, France.

Received: 26 November 2012 Accepted: 19 March 2013

Published: 11 April 2013

\section{References}

1. Wilson JW: Nocardiosis: updates and clinical overview. Mayo Clin Proc 2012, 87:403-407.

2. Brown-Elliott BA, Brown JM, Conville PS, Wallace RJ Jr: Clinical and laboratory features of the Nocardia spp. based on current molecular taxonomy. Clin Microbiol Rev 2006, 19:259-282.

3. Pijper A, Pullinger BD: South African nocardiases. J Trop Med Hyg 1927, 32:153-156

4. Wilson RW, Steingrube VA, Brown BA, Blacklock Z, Jost KC Jr, McNabb A Colby WD, Biehle JR, Gibson JL, Wallace RJ Jr: Recognition of a Nocardia transvalensis complex by resistance to aminoglycosides, including amikacin, and PCR-restriction fragment length polymorphism analysis. J Clin Microbiol 1997, 35:2235-2242.

5. Conville PS, Brown JM, Steigerwalt AG, Brown-Elliott BA, Witebsky FG: Nocardia wallacei sp. nov. and Nocardia blacklockiae sp. nov., human pathogens and members of the "Nocardia transvalensis Complex". J Clin Microbiol 2008, 46:1178-1184

6. Conville PS, Brown-Elliott BA, Wallace RJ Jr, Witebsky FG, Koziol D, Hall GS, Killian SB, Knapp CC, Warshauer D, Van T, Wengenack NL, Deml S, Woods GL: Multisite reproducibility of the broth microdilution method for susceptibility testing of Nocardia species. J Clin Microbiol 2012, 50:1270-1280.

7. Uhde KB, Pathak S, McCullum I Jr, Jannat-Khah DP, Shadomy SV, Dykewicz CA, Clark TA, Smith TL, Brown JM: Antimicrobial-resistant nocardia isolates, United States, 1995-2004. Clin Infect Dis 2010, 51:1445-1448. 
8. Larruskain J, Idigoras P, Marimón JM, Pérez-Trallero E: Susceptibility of 186 Nocardia sp. isolates to 20 antimicrobial agents. Antimicrob Agents Chemother 2011, 55:2995-2998.

9. Tremblay J, Thibert L, Alarie I, Valiquette L, Pépin J: Nocardiosis in Quebec, Canada, 1988-2008. Clin Microbiol Infect 2011, 17:690-696.

10. Drancourt M, Berger P, Raoult D: Systematic 16S rRNA gene sequencing of atypical clinical isolates identified 27 new bacterial species associated with humans. J Clin Microbiol 2004, 42:2197-2202.

11. CLSI: Susceptibility testing of mycobacteria, nocardia and other aerobic actinomycetes. CLSI document M24-A2. Wayne, PA: Clinical and Laboratory Standards Institute; 2011

12. Yorke RF, Rouah E: Nocardiosis with brain abscess due to an unusual species, Nocardia transvalensis. Arch Pathol Lab Med 2003, 127:224-226.

13. Brown-Elliott BA, Biehle J, Conville PS, Cohen S, Saubolle M, Sussland D, Wengenack N, Kriel K, Bridge L, McNulty S, Vasireddy R, Wallace RJ Jr: Sulfonamide resistance in isolates of Nocardia spp. from a US multicenter survey. J Clin Microbiol 2012, 50:670-672.

14. Lai C-C, Liu W-L, Ko W-C, Chen Y-H, Tang H-J, Huang Y-T, Hsueh P-R: Antimicrobial-resistant nocardia isolates, Taiwan, 1998-2009. Clin Infect Dis 2011, 52:833-835.

15. Hitti W, Wolff M: Two cases of multidrug-resistant Nocardia farcinica infection in immunosuppressed patients and implications for empiric therapy. Eur J Clin Microbiol Infect Dis 2005, 24:142-144.

doi:10.1186/1752-1947-7-103

Cite this article as: Cassir et al: Sulfonamide resistance in a

disseminated infection caused by Nocardia wallacei: a case report. Journal of Medical Case Reports 2013 7:103.

\section{Submit your next manuscript to BioMed Central and take full advantage of:}

- Convenient online submission

- Thorough peer review

- No space constraints or color figure charges

- Immediate publication on acceptance

- Inclusion in PubMed, CAS, Scopus and Google Scholar

- Research which is freely available for redistribution 